\title{
Computation and Analysis of Compliance in Grasping and Fixturing
}

\author{
Qiao Lin, Joel Burdick \\ Dept. of Mechanical Engineering \\ California Inst. of Technology \\ Pasadena, CA 91125
}

\author{
Elon Rimon \\ Dept. of Mechanical Engineering \\ Technion, Israel Inst. of Technology \\ Haifa, ISRAEL
}

\begin{abstract}
This paper presents a method to compute stiffness matrices for compliant grasps and fixtures. While the linear spring contact model has been widely used by robotics researchers, it is in general not accurate for practical applications. More realistic models, including the well-verified Hertz model, are incorporated by use of overlap functions. We derive a stiffness matrix formula that considers surface and material properties of the contacting bodies and applies to both planar and solid grasps. The effects of contact geometry are analyzed and illustrated with examples.
\end{abstract}

\section{Introduction}

This paper addresses the computation of stiffness matrices for compliant grasps and fixtures. Our method can incorporate quite general models of compliance, including the well known Hertz contact model, and takes the curvatures of the contacting bodies into account. The resulting stiffness matrices should be generally applicable to automated fixture and grasp planning. For the sake of convenience, the generic term grasping will also apply to fixturing in this paper.

Compliance can play a significant role in grasping and fixturing, and its analysis has received much attention. Among the first to study compliant grasps, Hanafusa and Asada [4] modeled each finger in a 3fingered planar grasp as a linear spring and showed how to find stable equilibrium grasps. Nguyen [12] also used a linear spring model to compute the stiffness matrix of more general grasps. He shows that the stiffness matrix depends on local curvature as well as the sticking or sliding of the fingers. Using linear spring modeling, Howard and Kumar [7] consider stability of planar grasps, while Donoghue et al. [2] investigate stable workpiece fixturing. Both studies develop stiffness matrix formulas that include the dependence on local contact geometry. Cutkosky and Wright [1] note that stability is influenced by initial loading as well as local curvature.

While the linear spring compliance model has been widely used in the robotics community, it is not supported by experiments or by results from elasticity theory. No systematic procedures have been proposed to position the linear spring elements that model contact compliance. Further, the linear spring stiffness coefficients must be determined from experiments, as there are no theoretical models to compute these coefficients from first principles. For automated fixture planning algorithms that would accurately compute fixturing arrangements, fixture geometries, fixture reaction forces, and fixtured object deflections from CAD models, these shortcomings of the linear spring model are significant.

Several researchers, such as Sinha and Abel [18], and Howard and Kumar [6] suggest an approach resembling finite elements. The contact regions are discretized into a number of small elements and elasticity theory is invoked to find the relative displacement of the contacting objects. While this approach is accurate, the complicated procedure has a number of drawbacks. First, the function giving force in terms of displacement (compliance relationship) can only be obtained numerically. Thus, the stiffness matrix, which is the differential of the compliance relationship, can only be found through complicated numerical methods. The compliance and stiffness functions are needed to compute quality measures that are used to find optimal grasping and fixturing arrangements [11]. Thus, these numerical approaches are not well suited to optimal fixture planning. Finally, the possibility of performing analysis on the stiffness matrix is lost with these numerical approaches.

A compliance model that is more accurate and more systematically deployable than the linear spring model is clearly needed. This model should afford a (nearly) closed form formula that is amenable to analysis and efficient computation. Rimon and Burdick [16] used overlap functions to develop lumped parameter models that can implement nonlinear compliance effects. In this paper, which extends their work, we show how to formulate the widely verified and theoretically justified Hertz contact model using overlap functions. The Hertz compliance function is then applied to the computation and analysis of the grasp stiffness matrix. Since the Hertz contact compliance function and stiffness matrix can be calculated from first principles, no experimental data are required for its use. Hence, our approach is attractive for automated planning algorithms. Further, our Hertzian stiffness matrix can be computed in a tractable symbolic form, which allows us to analyze the effects of contact geometry on stability, as well as the relative contribution of "first" and "second" order effects to overall grasp stiffness. Second order effects have recently been suggested as a means to reduce the number of fingers needed to fixture an object [14]. An 
example shows that these second order effects can be practically important.

\section{Background}

A grasp or fixturing arrangement consists of an object $\mathcal{B}$ contacted by $m$ fingers $\mathcal{A}_{1}, \ldots, \mathcal{A}_{m}$. We assume that the contacts are frictionless, and that the boundaries of the bodies near the contact points are smooth. We also assume that the bodies are quasi-rigid, and that $\mathcal{A}_{i}$ are stationary. In the quasi-rigid assumption, deformations due to compliance effects are assumed to be localized to the vicinity of the contact points, so that the overall motion of $\mathcal{B}$ relative to $\mathcal{A}_{i}$ can be described using rigid body kinematics. Since the fingers are stationary, we can focus on $\mathcal{B}$ 's configuration space (c-space), denoted by $\mathcal{C}$, which is the collection of all possible configurations of $\mathcal{B}$.

The c-space is a 6-dimensional manifold whose coordinates can be given as follows. Choose a fixed world reference frame, $\mathcal{F}_{W}$, and a frame $\mathcal{F}_{B}$ fixed to $\mathcal{B}$. The position, $d$, and orientation, $R \in S O(3)$, of $\mathcal{F}_{B}$ relative to $\mathcal{F}_{W}$ specify $\mathcal{B}$ 's location. The c-space can be parametrized by using the hybrid coordinates $q=(d, \theta) \in \mathbb{R}^{3} \times \mathbb{R}^{3}$ around a given configuration $\left(d_{0}, R_{0}\right)$ that has coordinates $q_{0}=\left(d_{0}, 0\right)$.

$$
d \mapsto d, \quad \theta \mapsto R(\theta)=\exp (\hat{\theta}) R_{0},
$$

where $\widehat{\theta}$ is a skew-symmetric matrix such that $\widehat{\theta} v=\theta \times v$ for $v \in \mathbb{R}^{3}$. The tangent space to $\mathcal{C}$ at $q_{0}$, denoted by $T_{q_{0}} \mathcal{C}$, is the set of all tangent vectors, or velocities of $\mathcal{B}$, at $q_{0}$. In hybrid coordinates, tangent vectors take the form $\dot{q}=\left(v^{T}, \omega^{T}\right)^{T}$, where $v \in \mathbb{R}^{3}$ is the velocity of the origin of $\mathcal{F}_{B}$, and $\omega \in \mathbb{R}^{3}$ is $\mathcal{F}_{B}$ 's angular velocity. Similarly, the wrench space at $q_{0}$ is the set of all wrenches acting on $\mathcal{B}$. Formally, this is the cotangent space of $\mathcal{C}$, denoted by $T_{q_{0}}^{*} \mathcal{C}$. A wrench takes the form $w=\left(f^{T}, \tau^{T}\right)^{T}$ in hybrid coordinates, where $f \in \mathbb{R}^{3}$ is a force acting at the origin of $\mathcal{F}_{B}$, and $\tau \in \mathbb{R}^{3}$ is a torque.

\section{Modeling Contact Compliance}

This section introduces the overlap representation of contact compliance and discuss its applicability. The Hertz contact theory is then reviewed.

\subsection{The Overlap Representation}

We wish to ignore the details of compliant surface deformations due to a relative displacement of contacting bodies, and model the resultant contact force as a function of the relative displacement. That is, we seek a lumped-parameter representation of the interaction between two quasi-rigid bodies. Rimon and Burdick [16] have shown that this can be achieved using overlap functions. Consider a single contact of $\mathcal{B}$, by a finger $\mathcal{A}$. We denote by $\mathcal{B}(q)$ the subset of $\mathbb{R}^{3}$ occupied by the undeformed shape of $\mathcal{B}$ at configuration $q$. Let the bound- aries of $\mathcal{B}(q)$ and the undeformed shape of $\mathcal{A}$ be denoted $\partial \mathcal{B}(q)$ and $\partial \mathcal{A}$, respectively. Rather than solve for the complex surface deformations that arise during compliant interaction, imagine that the rigid body shapes could freely interpenetrate without deformation during a relative approach. The overlap between $\mathcal{B}$ and $\mathcal{A}$, denoted $\delta$, is defined to be the minimum amount of translation that separates $\mathcal{B}$ from $\mathcal{A}$. In the absence of deformations $\mathcal{B}(q)$ and $\mathcal{A}$ can intersect at no more than an isolated point and we define $\delta=0$. It can be shown that there exist unique points $x \in \partial \mathcal{B}(q)$ and $y \in \partial \mathcal{A}$, as illustrated in Fig. 1, such that $\delta=\|x-y\|$. The normals to $\partial \mathcal{B}(q)$ and $\partial \mathcal{A}$ are collinear at these points: $n(x)=n(y)$. Note that $n(x)$ is inward-pointing and $n(y)$ is outward-pointing with respect to the respective boundaries.

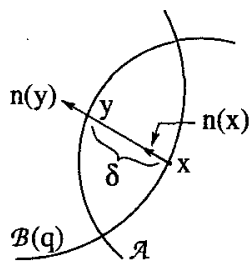

Fig. 1. The overlap model geometry

In the absence of deformations, the points $x$ and $y$ coincide and the bodies are in point contact. Thus, the overlap $\delta$ is precisely the relative approach of the bodies [3] as known in contact mechanics. The interactive forces are assumed to be collinear with the segment $\overline{x y}$ and have the magnitude $F=f(\delta)$. Clearly, the points $x$ and $y$ depend on $q$, the configuration of $\mathcal{B}$. Thus, the overlap $\delta$ is a function of $q: \delta=\delta(q)$. Moreover, as shown in [16], this function is differentiable when $\delta(q)>0$. Therefore, the finger force acting on $\mathcal{B}$ becomes a differentiable function of $q$, provided that $f(\delta)$ is so.

In 3.2 we will show that the overlap representation can be used to formulate the Hertz contact model. However, it is important to note that this representation is valid under more general circumstances. For example, the surfaces do not have to be smooth at the contact point, and the size of the contact area (described in 3.2) does not have to be always small compared with the size of the bodies, although in the former case $\delta(q)$ may fail to be differentiable. So long as the contacts are frictionless, and the relative approach of the elastic bodies is reasonably well-defined and remains small, the resultant contact force can be expressed as a function of the overlap. We acknowledge that when the Hertz model is not applicable, the determination of the functional relationship $f(\delta)$ can be difficult and there may exist no closed form formulations. Then finite elements may be used, or empirical models may be sought based on 
experimental data.

\subsection{The Hertz Contact Model}

We briefly summarize the Hertz contact model [5] [9], and formulate it using the overlap representation. In contact mechanics theory, a contact is said to be conforming if the surfaces of the bodies fit closely or even exactly in the absence of deformations. Bodies with dissimilar profiles are said to be non-conforming. When the two solids are brought into contact they touch initially at a single point (3D case) or a line (2D case for cylindrical bodies). Under the action of the load, they deform and touch over a finite area, termed contact area, surrounding the point or line of first contact. The interactive normal tractions in the contact area are called contact pressure.

Hertz theory considers the bodies $\mathcal{B}$ and $\mathcal{A}$ in nonconforming contact. The curvatures of $\partial \mathcal{B}(q)$ and $\partial \mathcal{A}$ at the contact point are given by the Weingarten maps [19] $L_{B}=-D n(x)$ and $L_{A}=D n(y)(n(x)$ and $n(y)$ are shown in Fig. 1), respectively, where $D$ is the differentiation operator. The relative curvature at the contact point is defined to be $L_{r e l}=L_{A}+L_{B}$, which is positive definite if the undeformed bodies are in point contact and $L_{A}$ and $L_{B}$ are not both zero. The eigenvalues of $L_{r e l}^{-1}$, called the principal radii of relative curvature, are denoted $r_{r e l 1}$ and $r_{r e l 2}\left(r_{r e l 1} \geqslant r_{r e l 2}\right)$. Hertz theory assumes that the boundary of the contact area is an ellipse with semi-axes $a$ and $b(a \geqslant b) . a$ and $b$ are assumed to be much smaller than the principal radii of relative curvature $r_{r e l 1}$ and $r_{r e l 2}$, the magnitude of the bodies' principal radii of curvature (the reciprocals of the eigenvalues of $L_{B}$ and $L_{A}$ ), and the characteristic dimension of the bodies.

An elegant relationship between the load and the relative approach can be found under the above assumptions for $3 \mathrm{D}$ contacts. The eccentricity ratio of the contact area, defined as $e=\left(1-\frac{b^{2}}{a^{2}}\right)^{\frac{1}{2}}$, is determined by

$$
\frac{\left(1-e^{2}\right)^{-1} \mathbf{E}(e)-\mathbf{K}(e)}{\mathbf{K}(e)-\mathbf{E}(e)}=\frac{r_{r e l 1}}{r_{r e l 2}}
$$

where $\mathbf{K}(e)$ and $\mathbf{E}(e)$ are complete elliptic integrals. Johnson [9] shows that if the eccentricity is not too large, this can be approximated by the simple relation

$$
\frac{a}{b}=\left(\frac{r_{r e l 1}}{r_{r e l 2}}\right)^{\frac{2}{3}}
$$

Let $r_{r e l}=\sqrt{r_{r e l 1} r_{r e l 2}}$. The contact force magnitude, the maximum pressure $p_{\max }$ over the contact area and $c=\sqrt{a b}$, which together with $e$ determines $a$ and $b$, are given as follows in terms of the overlap $\delta$.

$$
\begin{aligned}
f(\delta) & =(4 / 3) \beta_{1}(e) E^{*} r_{r e l} \frac{1}{2} \delta^{\frac{3}{2}}, \\
p_{m a x} & =(2 / \pi) \beta_{2}(e) E^{*}\left(\delta / r_{r e l}\right)^{\frac{1}{2}}, \\
c & =\beta_{3}(e)\left(r_{r e l} \delta\right)^{\frac{1}{2}},
\end{aligned}
$$

where $E^{*}$ is defined by $\frac{1}{E^{*}}=\frac{1-\nu_{A}^{2}}{E_{A}}+\frac{1-\nu_{B}^{2}}{E_{B}}$, with $E_{B}$ and $E_{A}$ Young's moduli, and $\nu_{A}$ and $\nu_{B}$ Poisson's ratios of $\mathcal{B}$ and $\mathcal{A}$, respectively. The coefficients $\beta_{1}-\beta_{3}$, which approach 1 as $e \rightarrow 0$, are given as follows.

$$
\begin{aligned}
& \beta_{1}(e)=\frac{\pi}{\sqrt{2} e \mathbf{K}(e)^{\frac{3}{2}}} \Phi(e)^{\frac{1}{4}}, \\
& \beta_{2}(e)=\frac{\pi e}{2 \sqrt{2}\left(1-e^{2}\right)^{\frac{1}{2}} \mathbf{K}(e)^{\frac{1}{2}}} \Phi(e)^{-\frac{1}{4}}, \\
& \beta_{3}(e)=\frac{\sqrt{2}\left(1-e^{2}\right)^{\frac{1}{2}}}{e \mathbf{K}(e)^{\frac{1}{2}}} \Phi(e)^{\frac{1}{4}}, \\
& \Phi(e)=(\mathbf{K}(e)-\mathbf{E}(e))\left(\left(1-e^{2}\right)^{-1} \mathbf{E}(e)-\mathbf{K}(e)\right) .
\end{aligned}
$$

In deriving Eqs. (3) -(5), each elastic body acted upon by the contact pressure is approximated by an infinite half-space of elastic material. This treatment, which can be justified by the smallness of the contact area, allows us to ignore the detailed shape of the body outside the local contact region. The displacement of the contact point is determined with reference to a point located infinitely far from the contact point.

The line contact problem can be considered as the limiting case of a point contact problem in which $a \rightarrow$ $\infty$. However, a tremendous complication occurs in this limiting process: relative to a point at infinity, the displacement of the contact point is no longer bounded and approaches infinity. Therefore, to obtain a finite displacement, the reference point must be located at a finite distance from the contact. Let us choose a reference point for $\mathcal{B}$ such that it is an interior point, at a distance $\ell_{B}$ from the contact point and on the line containing the contact normals. It can be shown [20] that the displacement of the contact point on $\mathcal{B}$ is

$$
\delta_{B}=\frac{\left(1-\nu_{B}^{2}\right) F}{\pi E_{B}}\left(2 \ln \frac{2 \ell_{B}}{b}-\frac{\nu_{B}}{1-\nu_{B}}\right),
$$

where $F$ is the contact force magnitude and $b=$ $\left(\frac{4 F r_{\text {rel }}}{\pi E^{*}}\right)^{\frac{1}{2}}$ is the half-width of the contact zone. Here $\frac{1}{r_{r e l}}=\frac{1}{r_{A}}+\frac{1}{r_{B}}>0$, where $r_{B}$ and $r_{A}$ are the radii of $\mathcal{B}$ and $\mathcal{A}$ at the contact (positive if convex). Likewise we can choose a reference point for $\mathcal{A}$. By replacing the subscript $B$ with $A$ in Eq. (6), we obtain the displacement $\delta_{A}$ of $\mathcal{A}$. The relative approach of $\mathcal{A}$ and $\mathcal{B}$ is thus:

$$
\delta=\delta_{A}+\delta_{B} .
$$

This equation determines the function $F=f(\delta)$. While there is in general no natural choice of reference points, the force-displacement relationship depends on the logarithm of distances $\ell_{A}$ and $\ell_{B}$. As noted by Johnson [8], this assures that $f(\delta)$ is reasonably insensitive to the choice of reference points.

These formulations of the load-overlap functional relationship are all nonlinear. While the linear spring model is generally not appropriate, there are special circumstances where a linear relationship can be approximately used. For a circular cylinder of finite length 
in non-conforming contact with a pair of diametrically opposed bodies, Nikpur and Gohar [13] review approximate formulas including linear or nearly linear spring relationships. In the context of robotic grasping, if contacts resemble this structure, the linear spring model can be used with reasonable accuracy. Otherwise, the linear spring model is generally not justified.

When the bodies are conforming, the Hertzian conditions are no longer satisfied. It is no longer adequate to consider only local deformations, and the overall deformation of at least one of the bodies must be considered. The determination of the functional relationship $F=f(\delta)$ becomes very difficult. In general this function is no longer in closed form and must be found numerically or experimentally. Here we mention the interesting results of Goodman and Keer [3]. They investigate the problem of an elastic sphere indenting an elastic spherical cavity, whose radii of curvature are only slightly larger than the sphere's. Their numerical results show a moderately harder load-overlap relationship than predicted by the Hertz model. It may therefore be possible to treat contacts of this type by the Hertz theory and obtain a conservative estimate of contact compliance.

\section{Computation of the Stiffness Matrix}

We first express the elastic potential as a function on the c-space. Let $\delta_{i}(q)$ be the overlap between $\mathcal{B}(q)$ and $\mathcal{A}$, and $f_{i}\left(\delta_{i}\right)$ be the finger force magnitude associated with a given compliance model (such as the Hertz model). Then the potential energy of the elastic system consisting of the object $\mathcal{B}$ and fingers $\mathcal{A}_{1}, \ldots, \mathcal{A}_{m}$ is

$$
\Pi(q)=\sum_{i=1}^{m} \int_{0}^{\delta_{i}(q)} f_{i}(\delta) d \delta .
$$

For the contacting bodies that are made of practical materials and modelled by Hertz theory, $f_{i}\left(\delta_{i}\right)$ are differentiable and $f_{i}^{\prime}\left(\delta_{i}\right)>0$. Hence $\Pi$ is differentiable at $q$ if $\delta_{i}(q)>0$, and we can differentiate $\Pi$ at an equilibrium configuration $q_{0}$ such that $\delta_{i}\left(q_{0}\right)>0$ for all $i$.

Consider a configuration $q_{0}$ of $\mathcal{B}$ such that $\mathcal{B}$ is in an equilibrium state (in the absence of any external wrench) under the action of some nonzero finger forces. This arrangement of fingers is said to be an equilibrium grasp at equilibrium configuration $q_{0}$. The nonzero finger forces are called preloading. When subjected to an arbitrary external disturbance, $\mathcal{B}$ may be displaced from $q_{0}$. The grasp is stable if $\mathcal{B}$ returns to $q_{0}$ after the external disturbance is removed. It is well-known that the gradient $\nabla \Pi\left(q_{0}\right)=0$ is necessary for equilibrium, and the Hessian $D^{2} \Pi\left(q_{0}\right)$ being positive definite is sufficient for stability.

Parameterize the c-space around $q_{0}$ using (1). Equi- librium implies that

$$
\nabla \Pi\left(q_{0}\right)=\sum_{i=1}^{m} f_{i}\left(\delta_{i 0}\right) \nabla \delta_{i 0}=0,
$$

where $\delta_{i 0}=\delta_{i}\left(q_{0}\right)$ and $\nabla \delta_{i 0}=\nabla \delta_{i}\left(q_{0}\right)$. Note that the preloading overlaps must be positive: $\delta_{i}\left(q_{0}\right)>0$. The gradient of $\delta_{i}$ is given by [16]:

$$
\nabla \delta_{i 0}=-\left(\begin{array}{c}
n_{i} \\
R_{0} r_{i} \times n_{i}
\end{array}\right) .
$$

Here $n_{i}=n\left(x_{i}\right)$, where $x_{i}$ is the initial point of contact (Fig. 1) for $\mathcal{B}\left(q_{0}\right)$ and $\mathcal{A}_{i}$, with $r_{i}$ the coordinates of $x_{i}$ with respect to the object frame.

A sufficiently small displacement of $\mathcal{B}$ can be approximated by a tangent vector at $q_{0}$. The new configuration is approximately $q_{0}+\dot{q} \Delta t$ for some $\dot{q} \in T_{q_{0}} \mathcal{C}$ and small scalar $\Delta t$. Hence, we will interchangeably use the terms velocity, tangent vector and displacement. To the same order of approximation, the restoring wrench resulting from the displacement is given by $K \dot{q} \Delta t$, where $K=D^{2} \Pi\left(q_{0}\right)$ is the Hessian of $\Pi$, and is called the stiffness matrix of the elastic system. With notation $f_{i}^{\prime}=\frac{d f_{i}}{d \delta_{i}}$ and $D^{2} \delta_{i 0}=D^{2} \delta_{i}\left(q_{0}\right)$, Eq. (7) yields

$$
K=\sum_{i=1}^{m}\left\{f_{i}^{\prime}\left(\delta_{i 0}\right) \nabla \delta_{i 0} \nabla \delta_{i 0}{ }^{T}+f_{i}\left(\delta_{i 0}\right) D^{2} \delta_{i 0}\right\} .
$$

As we will show, unlike the first summand in (10), the second summand involves the curvatures at the contacts. For this reason we call them first and second order terms, respectively. If the first order term in $K$ is positive definite, the grasp is said to be stable to the first order. If this is not the case but $K$ is stable because of the presence of the second order term, we say the grasp is stable to the second order.

In order to compute the stiffness matrix, we need to be able to compute $\delta_{i 0}$ and $D^{2} \delta_{i 0}$, with $\nabla \delta_{i 0}$ given in (9). We first consider the calculation of $\delta_{i 0}$, which can be divided into two cases: starting from either preloading finger forces or object displacement.

Consider specifying the preloading finger forces. Then by inverting the functions $f_{i}$ subject to the constraint (8), we can obtain $\delta_{i 0}$. Quite often, as is the case with generic 2-, 3- and 4-fingered planar grasps [15], [10], the specification of the sum of the finger forces $f_{T}=\sum_{i=1}^{m} f_{i}\left(\delta_{i 0}\right)$ uniquely determines the magnitude of individual finger forces. Then the preloading overlaps are functions of a single variable $f_{T}$.

We may also measure $\mathcal{B}$ 's configurations, denoted by $q_{\text {init }}$ and $q_{0}$, before and after preloading. Let $\Delta q=$ $q_{0}-q_{\text {init }}$ (written as a column vector), which is a displacement of the object. Then to the first order, the preloading overlaps are given by

$$
\delta_{i 0}=\nabla \delta_{i}\left(q_{\text {init }}\right)^{T} \Delta q
$$

where $\nabla \delta_{i}\left(q_{i n i t}\right)$ is given by (9), with $q_{0}$ replaced by $q_{\text {init }}$ and $R_{0}$ replaced by $R_{\text {init }}$, the orientation of $\mathcal{F}_{B}$ at $q_{i n i t}$. Note that $n_{i}$ and $r_{i}$ in Eq. (9) correspond to $q_{i n i t}$. 
We summarize the computation of $D^{2} \delta_{i 0}$, and refer to [10] for a proof. The subscript $i$ will be omitted for notational brevity. Recall that with respect to $\mathcal{F}_{W}$, $x_{0}=x\left(q_{0}\right) \in \partial \mathcal{B}\left(q_{0}\right)$ is an end point of the overlap $\delta\left(q_{0}\right)$ (Fig. 1). This point has coordinates $r_{0}=r\left(q_{0}\right)$ with respect to $\mathcal{F}_{B}$ determined by the relationship

$$
x\left(q_{0}\right)=R_{0} r\left(q_{0}\right)+d_{0} .
$$

Denote $n\left(x\left(q_{0}\right)\right)$ by $n_{0}$, which is the unit normal pointing into $\partial \mathcal{B}\left(q_{0}\right)$ at $x\left(q_{0}\right)$. Let $\overline{\mathcal{A}}$ be an imaginary (rigid) finger obtained by uniformly compressing the finger $\mathcal{A}$ by the amount $\delta\left(q_{0}\right)$. Then $\mathcal{B}$ and $\overline{\mathcal{A}}$ are in point contact at $x\left(q_{0}\right)$. Now, let $L_{B}\left(x\left(q_{0}\right)\right)$ be the Weingarten map of $\partial \mathcal{B}\left(q_{0}\right)$ at $x\left(q_{0}\right)$, and $L_{\bar{A}}\left(x\left(q_{0}\right)\right)$ be the Weingarten map of the boundary of the imaginary finger $\mathcal{A}$ at $x\left(q_{0}\right)$, both specified relative to $\mathcal{F}_{W}$. With arguments dropped from these maps for brevity, the relative curvature at the contact is defined by $\bar{L}_{r e l}=L_{B}+L_{\bar{A}}$.

Proposition 4.1. The $(1,1),(1,2)$ and $(2,2)$ entries of the symmetric matrix $D^{2} \delta\left(q_{0}\right)$ are given by

$$
\Phi_{11}=-\mathcal{L}_{\bar{A}} \bar{L}_{r e l}^{-1} \mathcal{L}_{B}, \quad \Phi_{12}=\mathcal{L}_{\bar{A}} \bar{L}_{r e l}^{-1}\left(\mathcal{L}_{B} \widehat{p}+\widehat{n}_{0}\right),
$$

$\Phi_{22}=\widehat{n}_{0}^{T} \bar{L}_{r e l}^{-1} \widehat{n}_{0}-\widehat{p}^{T} \mathcal{L}_{\bar{A}} \bar{L}_{r e l}^{-1} \mathcal{L}_{B} \widehat{p}+\left(\widehat{n}_{0} \bar{L}_{r e l}^{-1}\left(\mathcal{L}_{\bar{A}}-\mathcal{L}_{B}\right) \widehat{p}\right)_{S}$, where $\mathcal{L}_{\bar{A}}=M L_{\bar{A}} M, \mathcal{L}_{B}=M L_{B} M, M=I-n n^{T}$, $p=R_{0} r_{0}$, and $(\cdot)_{s}$ gives the symmetric part of a matrix.

For planar grasps, the $z$-axis is perpendicular to the plane. The equilibrium configuration is thus $\left(d_{0}, R_{0}\right)$ with $d_{0} \in \mathbb{R}^{2}$ and $R_{0} \in S O(2)$. A tangent vector takes the form $\dot{q}=\left(v^{T}, \omega\right)^{T}$ with $v \in \mathbb{R}^{2}$ and $\omega$ a scalar. The radii of curvature of the planar bodies $\mathcal{B}, \mathcal{A}$ and $\overline{\mathcal{A}}$ at the contact point are $r_{B}, r_{A}$ and $\bar{r}_{A}=r_{A}-\delta\left(q_{0}\right)$, respectively. Let $\rho=-R_{0} r\left(q_{0}\right) \cdot n_{0}$, and $\tilde{n}_{0}=R\left(-\frac{\pi}{2}\right) n_{0}$. Then Proposition 4.1 simplifies to

Corollary 4.2. In the planar case

$$
D^{2} \delta\left(q_{0}\right)=\frac{1}{\bar{r}_{A}+r_{B}}\left(\begin{array}{cc}
-\tilde{n}_{0} \tilde{n}_{0}^{T} & \left(r_{B}-\rho\right) \tilde{n}_{0} \\
\left(r_{B}-\rho\right) \tilde{n}_{0}^{T} & \left(\bar{r}_{A}+\rho\right)\left(r_{B}-\rho\right)
\end{array}\right) .
$$

\section{Effects of Contact Geometry}

In this section we consider the effects of contact geometry on the stability of a grasp. Sufficient conditions will first be given for the second order effects to be negligible compared with the first order effects. Then we study possible stabilizing and destabilizing effects of local contact geometry, and their importance relative to the first order effects.

Given a matrix $A$, its spectral norm is defined as $\|A\|=\sqrt{\lambda_{\max }\left(A^{T} A\right)}$. For two symmetric matrices $A$ and $B$, we write $A \succcurlyeq B, A \preccurlyeq B, A \succ B$ or $A \prec B$ if $A-B$ is positive semidefinite, negative semidefinite, positive definite, or negative definite, respectively.

For convenience in order-of-magnitude analysis, we nondimensionalize the stiffness matrix as follows. Let $\ell$ be a characteristic length of $\mathcal{B}$. For an $m$-fingered equilibrium grasp at configuration $q_{0}$, let $f_{T}=\sum_{j=1}^{m} f_{j}\left(\delta_{j 0}\right)$,
$\delta_{0}=f_{T} / k_{0}$, and $\nu_{i}=f_{i}\left(\delta_{i 0}\right) / f_{T}$, where $k_{0}$ is a (constant) characteristic contact stiffness that has the order of magnitude of $f_{i}^{\prime}\left(\delta_{i 0}\right)$. Note that $\delta_{0}$ has the same order of magnitude as $\delta_{i 0}$ and that the coefficients $\nu_{i}$ are not necessarily unique. Scale velocities and wrenches by $\dot{\tilde{q}}=S^{-1} \dot{q}$ and $\tilde{\boldsymbol{w}}=\frac{1}{k_{0}} S \boldsymbol{w}$, where $S=\operatorname{diag}\left(I, \frac{1}{\ell} I\right)$. The scaled stiffness matrix $\tilde{K}$ such that $\tilde{\boldsymbol{w}}=\tilde{K} \dot{\tilde{q}}$ is given by

$$
\tilde{K}=G D G^{T}+\sum_{i=1}^{m} \nu_{i} \Psi_{i},
$$

where $G=\left[g_{1}, \ldots, g_{m}\right]$ with $g_{i}=S \nabla \delta_{i 0}, D=$ $\operatorname{diag}\left(f_{1}^{\prime}\left(\delta_{i 0}\right) / k_{0}, \ldots, f_{m}^{\prime}\left(\delta_{i 0}\right) / k_{0}\right)$, and

$$
\Psi=\left(\begin{array}{ll}
\Psi_{11} & \Psi_{12} \\
\Psi_{12}^{T} & \Psi_{22}
\end{array}\right)=\left(\begin{array}{ll}
\delta_{0} \Phi_{11} & \frac{\delta_{0}}{\ell} \Phi_{12} \\
\frac{\delta_{0}}{\ell} \Phi_{12}^{T} & \frac{\delta_{0}}{\ell^{2}} \Phi_{22}
\end{array}\right)
$$

We observe that $\left\|G D G^{T}\right\|$ is of order 1: $\left\|G D G^{T}\right\| \sim$ 1 , and that the second order effects are insignificant compared with the first order effects if $\|\Psi\| \ll 1$, or equivalently, $\left\|\Psi_{i j}\right\| \ll 1$ for $i, j=1,2$.

It follows from Proposition 4.1 that when $\left\|L_{\bar{A}}\right\|,\left\|L_{B}\right\|$ and $\left\|\bar{L}_{r e l}^{-1}\right\|$ are bounded, the curvature effects are also bounded. Let us write $z=\mathcal{O}(a)$ if either $|z| \ll a$ or $|z| \sim a$ for $z, a \in \mathbb{R}$. The following proposition, whose proof appears in [10], gives a set of sufficient conditions for the second order effects to be insignificant.

Proposition 5.1. We have $\left\|\Psi_{i j}\right\| \ll 1$ for $i, j=1,2$ if the following conditions are satisfied.

1. $\delta_{0}\left\|L_{\bar{A}}\right\| \ll 1, \delta_{0}\left\|L_{B}\right\| \ll 1$, and $\delta_{0}\left\|\bar{L}_{r e l}\right\| \ll 1$.

2. $\frac{\left\|L_{\bar{A}}\right\|}{\left\|\bar{L}_{\text {rel }}\right\|}, \frac{\left\|L_{B}\right\|}{\left\|\bar{L}_{r e l}\right\|}$, and $\frac{\left\|\bar{L}_{\text {rel }}^{-1}\right\| / \ell}{\left\|\bar{L}_{\text {rel }}\right\| \ell}$ are $\mathcal{O}(1)$.

For concreteness, let us apply these conditions to a planar grasp, which yields the following corollary.

Corollary 5.2. For a planar grasp, the following conditions are sufficient for insignificant second order effects.

1. $\bar{r}_{A} \gg \delta_{0}, r_{B} \gg \delta_{0}$ and $\bar{r}_{r e l}=\frac{\bar{r}_{A} r_{B}}{\left(\bar{r}_{A}+r_{B}\right)} \gg \delta_{0}$.

2. $\frac{\bar{r}_{\text {rel }}}{\bar{r}_{A}}=\mathcal{O}(1), \frac{\bar{r}_{\text {rel }}}{r_{B}}=\mathcal{O}(1)$ and $\frac{\bar{r}_{\text {rel }}}{\ell}=\mathcal{O}(1)$.

We observe that the conditions $\delta_{0}\left\|L_{\bar{A}}\right\| \ll 1$ and $\delta_{0}\left\|L_{B}\right\| \ll 1$ are not restrictive at all. They require that the smallest radii of curvature at the contact point be much greater than $\delta_{0}$, which is satisfied by all contacts that are physically meaningful. Therefore, we will hereafter assume that these two conditions are always satisfied.

We now consider stabilizing and destabilizing effects of local geometry by looking at $\tilde{K}$. Provided $f_{i}^{\prime}\left(\delta_{i 0}\right)>0$, the matrix $G D G^{T}$ is positive semidefinite. Thus, the first order effects are stabilizing. To investigate the curvature effects on grasp stability, we focus on the matrix $\Psi_{i}$ associated with the $i^{\text {th }}$ contact and drop the index $i$ for brevity.

Recall that the positive definiteness of a partitioned matrix implies the positive definiteness of its diagonal blocks. The presence of $\bar{L}_{r e l}$, which is positive definite, in $\Psi_{22}$ indicates that this term can be rotationally stabilizing. The presence of $\mathcal{L}_{\bar{A}} \bar{L}_{r e l}^{-1} \mathcal{L}_{B}$ in both $\Psi_{11}$ and $\Psi_{22}$ 
means that this term can be stabilizing translationally and rotationally if it is negative semi-definite, and destabilizing if positive semi-definite. We state the following observations for four different contact types.

1. $L_{\bar{A}} \succcurlyeq 0$ and $L_{B} \succcurlyeq 0$ (convex vs. convex surfaces): $\mathcal{L}_{\bar{A}} \bar{L}_{\text {rel }}^{-1} \mathcal{L}_{B} \succcurlyeq 0$ and hence destabilizing.

2. $L_{\bar{A}} \succ 0$ and $L_{B} \preccurlyeq 0$, or $L_{\bar{A}} \preccurlyeq 0$ and $L_{B} \succ 0$ (strictly convex vs. concave surfaces): in this case $\mathcal{L}_{\bar{A}} \bar{L}_{r e l}^{-1} \mathcal{L}_{B} \preccurlyeq 0$ and stabilizing.

3. $L_{\bar{A}} \succcurlyeq 0$ and $L_{B}$ indefinite, or $L_{\bar{A}}$ indefinite and $L_{B} \succcurlyeq 0$ (convex vs. saddle surfaces): $\mathcal{L}_{\bar{A}} \bar{L}_{r e l}^{-1} \mathcal{L}_{B}$ is indefinite (mixed effects).

4. $L_{\bar{A}}$ and $L_{\mathrm{B}}$ both indefinite (saddle vs. saddle surfaces): $\mathcal{L}_{\bar{A}} \bar{L}_{\text {rel }}^{-1} \mathcal{L}_{B} \prec 0$, stabilizing.

The second order effects may be stabilizing or destabilizing. A question that naturally arises is how significant these stabilizing or destabilizing effects can be as compared with the first order effects. To this end consider the decomposition $\Psi=\Psi_{a}+\Psi_{b}$ where

$$
\begin{aligned}
& \Psi_{a}=\left(\begin{array}{cc}
\delta_{0} \mathcal{L}_{\bar{A}} \bar{L}_{r e l}^{-1} \mathcal{L}_{\bar{A}} & \frac{\delta_{0}}{\ell} \mathcal{L}_{\bar{A}} \bar{L}_{r e l}^{-1} \mathcal{L}_{S} \\
\frac{\delta_{0}}{\ell} \mathcal{L}_{s}{ }^{T} \bar{L}_{r e l}^{-1} \mathcal{L}_{\bar{A}} & \frac{\delta_{0}}{\ell^{2}} \mathcal{L}_{S}{ }^{T} \bar{L}_{r e l}^{-1} \mathcal{L}_{S}
\end{array}\right), \\
& \Psi_{b}=\left(\begin{array}{cc}
-\delta_{0} \mathcal{L}_{\bar{A}} & 0 \\
0 & \frac{\delta_{0}}{\ell^{2}}\left(-\widehat{p}^{T} \mathcal{L}_{B} \widehat{p}+\left(\widehat{n}_{0} \widehat{p}\right)_{S}\right)
\end{array}\right),
\end{aligned}
$$

where $\mathcal{L}_{S}=\mathcal{L}_{B} \widehat{p}+\widehat{n}_{0}$. It is easy to see that $\Psi_{a}$ is positive semidefinite and hence is always stabilizing. Any possibly destabilizing curvature effects come from $\Psi_{b}$. Noting that $\delta_{0} / \ell \ll 1$ and $\|p\| / \ell \sim 1$, the following proposition can be shown straightforwardly.

Proposition 5.3. Provided that $\delta_{0}\left\|L_{\bar{A}}\right\| \ll 1$ and $\delta_{0}\left\|L_{B}\right\| \ll 1$, the destabilizing curvature effects, if any, are guaranteed to be very small. That is, $\left\|\Psi_{b}\right\| \ll 1$. On the other hand, if $\left\|\bar{L}_{\text {rel }}\right\| \ell \sim \frac{\delta_{0}}{\ell}$, then $\left\|\Psi_{a}\right\| \sim 1$ and the stabilizing curvature effects are significant.

It follows that the second order effects can never make a grasp that is stable to the first order unstable. Rather, they can be appropriately used so as to stabilize an otherwise unstable grasp, even with quite significant stabilizing effects. As $\left\|\bar{L}_{r e l}\right\|$ decreases (as the contacting surfaces achieve a better match), the stabilizing second order effects become quite significant. In particular, when the two surfaces fit sufficiently closely, the stabilizing second order effects can become comparable with first order effects. In [15] it was shown that curvature effects could be used to reduce the number of fixtures needed to immobilize, or fixture, an object. This analysis shows that by proper selection of the fixtures' curvature, fixtures with fewer contacts can still be sufficiently stiff.

The following corollary applies to a planar grasp. We replace $\bar{r}_{A}$ with $r_{A}$ when it is correct to do so.

Corollary 5.4. For a planar grasp, if $\delta_{0} /\left|r_{A}\right| \ll 1$ and $\delta_{0} /\left|r_{B}\right| \ll 1$, then any possibly destabilizing curvature effects are very small. For a contact between two non- concave surfaces, the second order stabilizing effects are significant if $r \delta_{0} / \ell^{2} \sim 1$ where $r=\min \left\{r_{A}, r_{B}\right\}$. For $a$ contact of a concave surface by a convex surface, these stabilizing effects are significant if $\left(\frac{r}{\ell}\right)^{2}\left(\frac{\delta_{0}}{\Delta r}\right) \sim 1$, where $r=\left|r_{B}\right|$ and $\Delta r=\left|r_{A}+r_{B}\right| \ll r$.

\section{Examples}

We present two three-fingered planar grasps to illustrate the effects of contact geometry. Choose coincident world and object frames and let the origin coincide with the concurrency point of the contact normals. Using convex spherical fingers contacting planar or concave cylindrical object surfaces, we employ the 3D Hertz model to find the compliance function $f(\delta)$ and use the planar formulas to compute the stiffness matrices.

We compare grasp stiffness as the finger tip radius varies by assuming the same level of preloading for different finger radii. At a contact the maximal shear stress [20] is approximately $\tau_{\max }=0.31 p_{\max }$ (see [9]). We preload the grasp to the capacity determined by $\tau_{m a x}=\gamma \sigma_{a}$ where $\sigma_{a}$ is the allowable stress of the material and $0<\gamma<1$. This gives a desired value of $p_{\max }$, and further determines the necessary preloading overlap $\delta_{0}$ by (4).

In each of the following two examples all three contacts are symmetrically arranged and have identical local geometry. Let $k_{0}=E^{*} \ell$ where $E^{*}=\frac{E}{2\left(1-\nu^{2}\right)}$ (all bodies are assumed to be made of the same material). Then the scaled stiffness matrix (11) can be written as $\tilde{K}=\tilde{k}\left\{\operatorname{diag}(I, 0)+\operatorname{diag}\left(\xi_{T} I, \xi_{R}\right)\right\}$

where

$$
\tilde{k}=3 \beta_{1}(e) \tilde{r}_{r e l} \sqrt{\mu}, \quad \mu=\left(\frac{\pi \gamma \sigma_{a}}{0.62 \beta_{2}(e) E^{*}}\right)^{2},
$$

with $\tilde{r}_{r e l}=r_{r e l} / \ell$. We may interpret $\tilde{k}$ as the dimensionless translational stiffness due to the first order effects. The scalars $\xi_{T}$ and $\xi_{R}$, which will be given in the individual examples, are a relative measure of contributions by the curvature effects.

Example 6.1. Consider a grasp of an equilateral triangular cylinder by three spherical fingers having uniform radius $r$ (Fig. 2). In this case $r_{r e l}=r$ and $e=0$, hence $\beta_{i}(e)=1$. Using the Hertz model and Corollary 4.2, we find

$$
\xi_{T}=0, \quad \xi_{R}=\frac{4}{3} \mu \tilde{r}(\tilde{r}+\tilde{\rho}) .
$$

Thus, while the grasp is not stable by considering only the first order effects, it is indeed second order stable. As $\tilde{r}$ increases, so do $\xi_{R}$ and $\tilde{k}$, since $\tilde{k}=3 \sqrt{\mu} \tilde{r}$. That is, both the first and second order effects become more significant as $\tilde{r}$ increases. Since $\sigma_{a} / E \sim 1 / 1000$, the curvature effects are relatively less significant. Even so, it is important to realize that they may be adequate for many practical applications.

Example 6.2. As shown in Fig. 3, a cylinder with three circular faces of uniform radius $r$ is grasped by three 


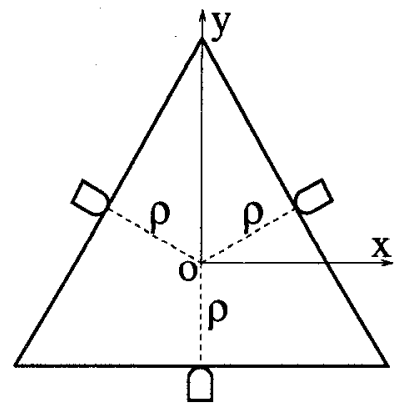

Fig. 2. Straight triangle grasp

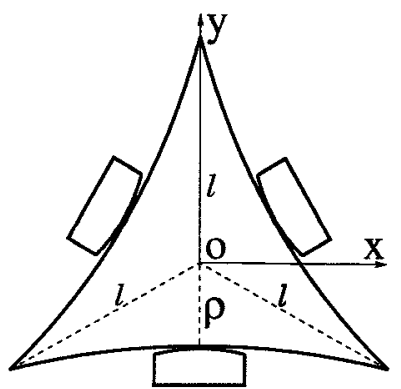

Fig. 3. Curved triangle grasp spherical fingers of radius $r-\Delta r(0<\Delta r \ll r)$. There are three axes of symmetry $\left(120^{\circ}\right.$ apart $)$ and the fingers are on these lines. Let $\epsilon=\frac{\Delta r}{r}, \tilde{r}=\frac{r}{\ell}$ and $\tilde{\rho}=\frac{\rho}{\ell}$. It can be shown that $\tilde{\rho}=\frac{1}{2}\left(\sqrt{4 \tilde{r}^{2}-3}-2 \tilde{r}+1\right)$. From Section 3.2, $r_{r e l 1}=\frac{r}{\epsilon}$ and $r_{r e l 2}=r$. Hence $\tilde{r}_{r e l}=\frac{\tilde{r}}{\sqrt{\epsilon}}$ and $e$ can be determined using (2). The Hertz model and Corollary 4.2 give

$$
\xi_{T}=\frac{2 \mu}{3(\epsilon \sqrt{\epsilon}+\mu)}, \quad \xi_{T}=\frac{4 \mu(\tilde{r}+\tilde{\rho})^{2}}{3(\epsilon \sqrt{\epsilon}+\mu)} .
$$

The ratios $\frac{\delta_{0}}{\Delta r}$ and $\frac{a}{r}$, where $a$ is the major semi-axis of the contact area, are given by

$$
\delta_{0} / \Delta r=\mu \epsilon^{-\frac{3}{2}}, \quad a / r=\beta_{3}(e) \mu^{\frac{1}{2}} \epsilon^{-\frac{1}{2}}\left(1-e^{2}\right)^{-\frac{1}{4}} .
$$

If we use steel, $E=207 \mathrm{GPa}, \nu=0.3$, and $\sigma_{a}=$ $140 \mathrm{MPa}$. Set the preload factor $\gamma=0.6$. The dependence of $\xi_{R}$ on $\tilde{r}$, and the curvature effect indicator $\lambda=\left(\frac{r}{\ell}\right)^{2}\left(\frac{\delta_{0}}{\Delta r}\right)=\frac{\mu \tilde{r}^{2}}{\epsilon \sqrt{\epsilon}}$ given in Corollary 5.4 shows that a large value of $\tilde{r}$ is advantageous. We choose $\tilde{r}=15$.

The parameters discussed above are tabulated in Table 1 for different values of $\epsilon$. The small values of $\phi=\arcsin (a / r)$ indicates the small size of the contact area and excellent accuracy of the Hertz model. As $\epsilon$ decreases, $\tilde{k}$ increases and hence the overall stiffness is enhanced. Meanwhile, $\xi_{R}$ is of order 1 as a result of decreasing $\epsilon$. Therefore, as the contacting surfaces approach a good match, the curvature effects can be very significant, as predicted by Corollary 5.4.

TABLE 1. First AND Second Order EFfects

\begin{tabular}{|c|c|c|c|c|c|c|}
\hline$\epsilon$ & $\lambda$ & $\bar{k}$ & $\xi_{T}$ & $\xi_{R}$ & $\delta_{0} / \Delta r$ & $\phi$ \\
\hline 0.01 & 1.091 & 1.422 & 0.0032 & 1.541 & 0.0048 & $1.2^{\circ}$ \\
\hline 0.02 & 0.492 & 1.039 & 0.0015 & 0.696 & 0.0022 & $0.9^{\circ}$ \\
\hline 0.03 & 0.306 & 0.865 & 0.0009 & 0.434 & 0.0014 & $0.8^{\circ}$ \\
\hline 0.04 & 0.218 & 0.759 & 0.0006 & 0.309 & 0.0010 & $0.8^{\circ}$ \\
\hline 0.05 & 0.167 & 0.686 & 0.0005 & 0.237 & 0.0007 & $0.7^{\circ}$ \\
\hline
\end{tabular}

\section{Conclusion}

While the linear spring model has been widely used by robotics researchers, it is not accurate or systematically applicable for automated planning procedures. In this paper we developed a stiffness matrix formula that includes both contact curvature effects and quite general nonlinear compliance models, including the widely verified Hertz contact model. We believe that these results will enable efficient and more accurate algorithms for automated planning of compliant grasps or fixtures. Our results also allow us to assess the relative contribution of contact geometry to grasp stability and effectiveness.

\section{References}

[1] M. Cutkosky and P. Wright. Friction, stability and the design of robotic fingers. Int. J. of Robotic Research, 5(4):20-37, 1986.

[2] J. P. Donoghue, W. S. Howard, and V. Kumar. Stable workpiece fixturing. In Proc. ASME Design Technical Conferences, Minneapolis, MN, Sept. 1994.

[3] L. E. Goodman and L. M. Keer. The contact stress problem for an elastic sphere indenting an elastic cavity. Int. J. of Solids Structures, 1(4):407-415, 1965.

[4] H. Hanafusa and H. Asada. Stable prehension by a robot hand with elastic fingers. In Proc. $7^{\text {th }}$ Int. Symp. on Industrial Robots, pages 361-368, Tokyo, October 1977.

[5] H. Hertz. On the contact of rigid elastic solids and on hardness. In Misc. Papers by H. Hertz. Jones and Schott, Macmillan, London, 1896. English translation.

[6] W. S. Howard and V. Kumar. A minimum principle for the dynamics analysis of systems with frictional contacts. In Proc. Int. Conf. on Robotics and Automation, pages 437442, Atlanta, GA, May 1993.

[7] W. S. Howard and V. Kumar. Stability of planar grasps. In Proc. Int. Conf. on Robotics and Automation, pages 28222827, San Diego, CA, May 1994.

[8] K. L. Johnson. One hundred years of hertz contact. Proc. Inst. Mechanical Engineers, 196:363-378, 1982.

[9] K. L. Johnson. Contact Mechanics. Cambridge University Press, 1985.

[10] Q. Lin, J. W. Burdick, and E. Rimon. Issues in optimal grasping and fixturing of workpieces. Technical report, Dept. of Mechanical Engineering, California Inst. of Technology, Pasadena, CA, 1996.

[11] Q. Lin, J. W. Burdick, and E. Rimon. A quality measure for compliant grasps. Submitted, IEEE Int. Conf. on Robotics and Automation, 1996.

[12] V.-D. Nguyen. Constructing stable grasps. Int. J. of Robotic Research, 8(1):26-37, 1989.

[13] K. Nikpur and R. Gohar. Deflexion of a roller compressed between platens. Tribology International, 8(2):2-8, 1975.

[14] E. Rimon and J. Burdick. New bounds on the number of frictionless fingers required to immobilize planar objects. $J$. Robotic Systems, 12(6):433-451, 1995.

[15] E. Rimon and J. W. Burdick. Mobility of bodies in contacti: A new $2^{\text {nd }}$ order mobility index for multiple-finger grasps. In Proc. Int. Conf. on Robotics and Automation, pages 23292335, San Diego, CA, May 1994.

[16] E. Rimon and J. W. Burdick. Mobility of bodies in contactii: How forces are generated by curvature effects. In Proc. Int. Conf. on Robotics and Automation, pages 2336-2341, San Diego, CA, May 1994.

[17] E. Rimon and J. W. Burdick. On force and form closure for multiple finger grasps. In Proc. Int. Conf. on Robotics and Automation, pages 1795-1800, May 1996. Minneapolis, MN.

[18] P. R. Sinha and J. M. Abel. A contact stress model for multifingered grasps of rough objects. IEEE Trans. on Robotics and Automation, 8(1):7-22, 1992.

[19] J. A. Thorpe. Elementary Topics in Differential Geometry. Spring-Verlag, 1979.

[20] S. Timoshenko. Strength of Materials. Van Nostrand, New York, 3rd edition, 1955. 